\title{
Infected kidney stone progressing to perinephric abscess and thoracic empyema
}

\author{
Antonio Tufano ${ }^{1}$, Rocco Minelli ${ }^{2}$, Giovanni Di Lascio ${ }^{1}$, Giampaolo Delicato ${ }^{3}$, Giulio Baffigo ${ }^{3}$, \\ Stefano Signore ${ }^{3}$ \\ ${ }^{1}$ Department of Maternal-Child and Urological Sciences, Sapienza Rome University, Policlinico Umberto I Hospital, Rome, Italy; \\ 2 Department Life and Health "V. Tiberio", University of Molise, Campobasso, Italy; \\ ${ }^{3}$ Department of Urology, Sant'Eugenio Hospital, Rome, Italy.
}

\section{Summary A case is presented that demonstrates unusual long-term evolution of an infected calculi, culminating in the formation of a retroperitoneal abscess that fistulised to the pleural space, leading to a right pleural empyema.}

KEY WORDS: Infected kidney stone; Perinephric abscess; Empyema.

Submitted 26 January 2020; Accepted 29 February 2020

\section{INTRODUCTION}

Perinephric and renal abscesses are uncommon, but potentially lethal complications of urinary tract infection. A perinephric abscess is usually a complication of an urologic infection (1). A perinephric abscess affects the renal capsule and Gerota's fascia. These abscesses can extend from the Gerota's fascia into the psoas and transversalis muscles as well as the peritoneal cavity and the pelvis (2). Renal calculi may harbour infection and cause localized obstruction. Urinary tract obstruction is generally considered a predisposing factor to urinary tract infection (3). Ascending urological infections towards the pleura are rare and only few cases are reported (4). Here we describe a case of a 63 year-old woman with infected staghorn calculi of the right kidney who developed a perinephric abscess that extended across the diaphragm to cause an empyema as well.

\section{Case report}

A 63 year-old women with a known history of recurrent urinary tract infections, including right-sided pyelonephritis presented because of fever $\left(39^{\circ} \mathrm{C}\right)$. She was normotensive but tachycardic (130/min) and in respiratory distress. Clinical examination showed signs of a large right pleural effusion but no abdominal tenderness. There was marked peripheral leucocytosis associated with a raised $C$-reactive protein (CRP).

Imaging, including computed tomography (CT) of thorax and abdomen, showed extensive peri-bronchial thickening, right lower lobe consolidation and a complex right pleural effusion with thickened pleura (Figires 1, 2).

In the right subdiaphragmatic area, a small hypodense collection of about 3 centimeters in diameter was appreciated in contiguity with the diaphragmatic dome.
The right kidney presents multiple hypodense areas of probable cystic nature and stones in thr middle and lower calyces and in the renal pelvis. The right kidney also demonstrated atrophic calcification, likely related to previous infections (Figures 3, 4).

A 24F intercostal catheter was urgently inserted with removal of $500 \mathrm{~mL}$ of turbid and exudative fluid which was cultured with growth of Citrobacter koseri. Subsequently a 8F nephrostomy catheter was placed in the right kidney with aspiration of purulent fluid positive for Citrobacter. Sensitivities to antibiotics were obtained and therapy with ceftazadime and meropenem was initiated obtaining a clinical improvement with reduction of fever and of the perirenal abscess size $(2 \mathrm{~cm})$. Evaluation of renal function by dynamic scintigraphy with 99mTc-DTPA showed right kidney failure. An open right radical nephrectomy was then performed and subsequently cardiothoracic surgeons proceeded with Video Assisted Thorascopic Surgery (VATS) to prevent the formation of a fibrothorax.

\section{Conclusions}

Perinephric abscesses can cause severe illness.

The severity of the disease depends on the extent of the infection and the comorbidities of the patient.

Infection of the renal and perinephric space continues to be a serious urological problem with high mortality rate. A high index of suspicion, prompt diagnosis, appropriate antibiotics and surgical intervention may be effective in reducing mortality.

\section{References}

1. Thorley JD, Jones SR, Sanford JP. Perinephric abscess. Medicine. 1974; 53441-451.

2. Sheinfeld J, Erturk E, Spataro RF, \& Cockett ATK. Perinephric Abscess: Current Concepts. The Journal of Urology. 1987; 137:191194.

3. Bichler KH, Eipper E, Naber K, et al. Urinary Infection stones. Int J Antimicrob Agents. 2002;19:488-98.

4. Stewart ZE, Shaker M, and David Baxter J. Urinary tract infection caused by Citrobacter koseri in a patient with spina bifida, an ileal conduit and renal calculi progressing to peri-nephric abscess and empyema. Urol. Case Rep. 2017; 11:22-24. 

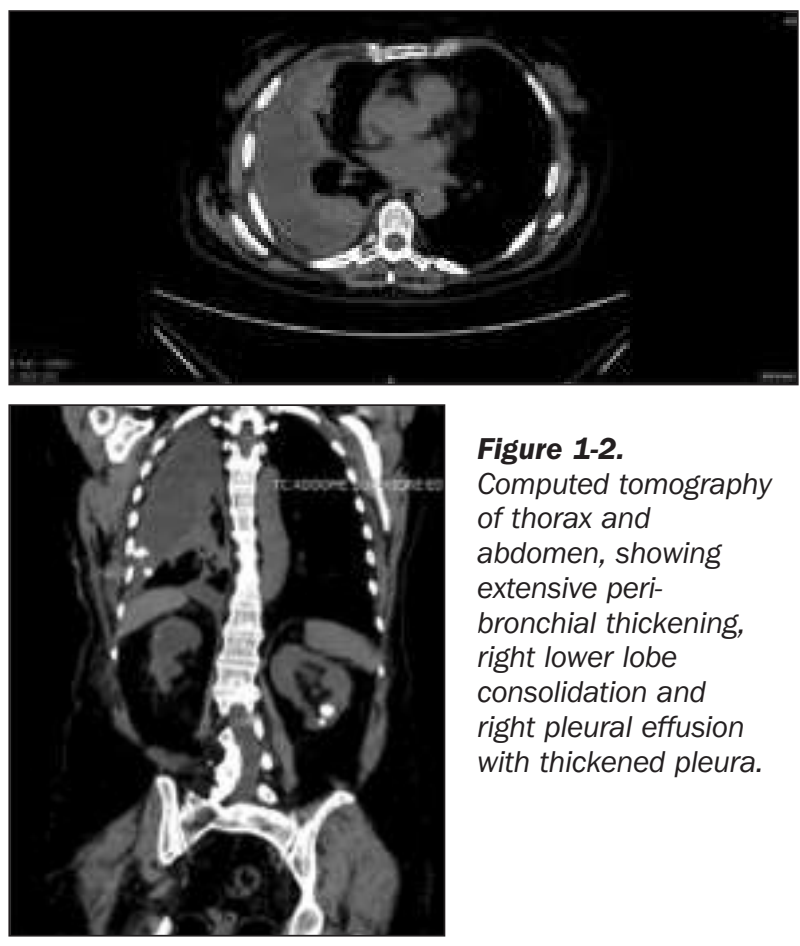

Figure 1-2.

Computed tomography of thorax and abdomen, showing extensive peribronchial thickening, right lower lobe consolidation and right pleural effusion with thickened pleura.

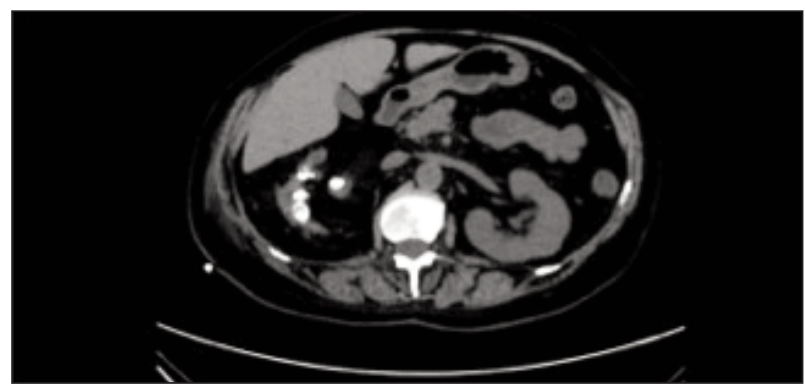

Figure 3-4.

TC showing right kidney atrophy with calcifications.

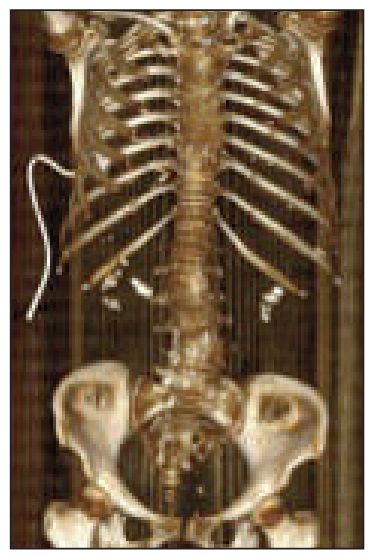

\section{Correspondence}

Antonio Tufano, MD

antonio.tufano91@gmail.com

Giovanni Di Lascio, MD

Department of Maternal-Child and Urological Sciences, Sapienza Rome University, Policlinico Umberto I Hospital

Viale del Policlinico 155, 00161 Rome (Italy)

\section{Rocco Minelli, MD}

Department Life and Health "V. Tiberio", University of Molise,

Via Francesco De Sanctis 1, Campobasso 86100 (Italy)

\section{Giampaolo Delicato, MD}

Giulio Baffigo, MD

Stefano Signore, MD

Department of Urology, Sant'Eugenio Hospital, Rome (Italy) 